The Frontline Reports column features short descriptions of novel approaches to mental health problems or creative applications of established concepts. Material should be 350 to 750 words long. Send material to Francine Cournos, M.D., at the New York State Psychiatric Institute (fc15@columbia.edu) or to Stephen M. Goldfinger, M.D., at SUNY Downstate Medical Center (smgoldfingermd@aol.com).

\section{Web-Based Intensive Therapeutic Contact for Eating Disorders}

Although many people suffer from eating disorders, which have high mortality and severe morbidity, only a small number of affected people in the Netherlands are treated by mental health professionals. Eating disorders are often not recognized. Many patients do not ask for help because of shame, a lack of awareness, or denial of the disorder. Even when the disorder is recognized, it is difficult to motivate patients to seek help. As a result, many patients are in urgent need of help.

To reach and treat more patients with eating disorders, Tactus Addiction Treatment developed a Web-based treatment program that uses intensive therapeutic contact. Such a program has already been shown to be a suitable medium for effective interventions for patients with alcohol abuse, depression, and anxiety disorders. Various interventions for patients with eating disorders are available online, but they primarily consist of guided self-help interventions. For some patients this form of treatment is effective, but intensive treatment with the support of a therapist is frequently needed. Therefore, personal support of a therapist is integrated as a main element in our Web-based intervention. During the program, patients communicate with their personal therapists twice a week. To keep the threshold as low as possible, the communication occurs asynchronously and via the Internet. This means that patients can participate in their personal environment at any time they prefer. Patients may also request an in-person or telephone contact supplementary to the online contacts. The therapist then makes an appointment to call the patient, or the patient is invited to come to the treatment location. Another unique characteristic of the program is that it is targeted to patients with all types of eating disorders. The information and all assignments are adjusted to the individual patient.

The Web-based intervention is based on the principles of cognitivebehavioral therapy and motivational interviewing. In part 1, patients analyze their eating attitudes and behaviors. Part 2 focuses on behavioral change. During the program, patients receive messages, assignments, exercises, and psychoeducation from their therapists. The program includes four assignments and at least seven contacts in part 1 and six assignments and at least 14 contacts in part 2. Examples of part 1 assignments are daily registering of eating behavior and related thoughts and feelings, analyzing eating situations, and describing advantages and disadvantages of the disorder. Part 2 assignments include setting goals in regard to eating, exercising, checking weight, and (if applicable) compensation behaviors, changing thoughts, changing behaviors, improving self-image, and writing a relapse prevention plan. The average duration of the program is 15 weeks. After completing the program, patients can participate in an aftercare program consisting of six weekly sessions.

In June 2009, our intervention was first tested in a population of 28 patients with eating disorders who were recruited via the Web site of a Dutch patient organization. The main aim of this pilot study was to evaluate patients' satisfaction and program feasibility. Initial effectiveness data were also measured. In addition to the regular online research questionnaires, the researcher interviewed each patient by telephone. The results of the pilot study were positive and suggested that the program was an acceptable and feasible intervention for patients with eating disorders. Several suggestions for improvement were received and implemented. Based on the positive results, in January 2010 the program was made widely available via the Internet for all patients with eating disorders. To highlight the intervention and to recruit patients, a nationwide press statement was released, and relevant health care institutions were informed about the intervention.

As a result, a total of 165 patients started the Web-based intervention in 2010. To evaluate the program, all patients were asked to complete online self-report measures at baseline, posttreatment, and six-week and six-month follow-up. Results showed that the Webbased intervention successfully changed eating disorder psychopathology, with most of the improvements sustained at the six-month follow-up. Physical and mental health also improved, and patients were much more satisfied with their body image. The patients evaluated the support of the therapist as most important, confirming our assumptions at the start of program development. To prove the efficacy of the program, we started a randomized controlled trial in March 2011. We expect those results to be available in January 2014.

Because of the wide availability of the Internet, our successful intervention is accessible for all patients with eating disorders in the Netherlands. We think it can easily be transformed to a worldwide intervention by translating the program to other languages.

Elke D. ter Huurne, M.Sc. Marloes G. Postel, Ph.D.

Cor A. J. De Jong, M.D., Ph.D.

Ms. ter Huurne and Dr. Postel are affiliated with Tactus Addiction Treatment, Institutenweg 17521 PH Enschede, the Netherlands (e-mail: e.terhuurne@tactus.nl). Ms. ter Huurne is also with Nijmegen Institute for Scientist Practitioners in Addiction, Nijmegen, the Netherlands, where Dr. De Jong is affiliated. Dr. Postel is also with the Department of Psychology Health and Technology, University of Twente, Enschede, the Netherlands. Dr. De Jong is also with the Behavioural Science Institute, Radboud University Nijmegen. 